\title{
Effects of a Greek Traditional Dance Programme on Sensorimotor Synchronization and Auditory Reaction Time of Young Children
}

\author{
Loukia Kapodistria a , Dimitris Chatzopoulos ba, , Katerina Chomoritic, \\ Georgios Lykesas ${ }^{d}$, Afroditi Lola ${ }^{e}$
}

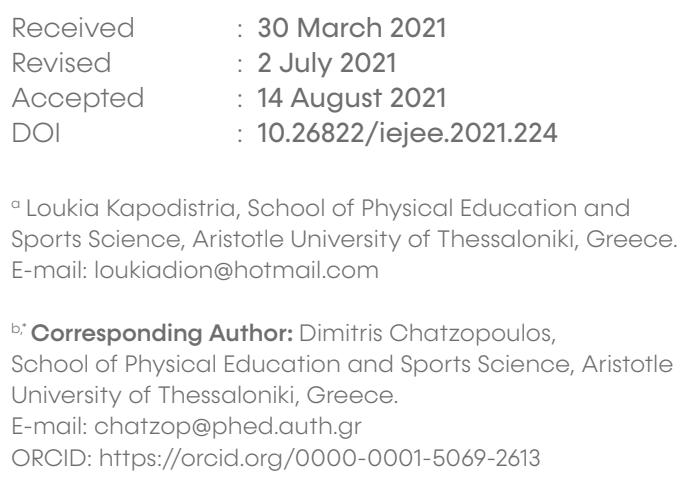

- Loukia Kapodistria, School of Physical Education and Sports Science, Aristotle University of Thessaloniki, Greece. E-mail: loukiadion@hotmail.com

\section{5: Corresponding Author: Dimitris Chatzopoulos,}

School of Physical Education and Sports Science, Aristotle University of Thessaloniki, Greece.

E-mail: chatzop@phed.auth.gr

ORCID: https://orcid.org/0000-0001-5069-2613

- Katerina Chomoriti, School of Physical Education and Sports Science, Aristotle University of Thessaloniki, Greece. E-mail: katerina_chomo@yahoo.gr

a Georgios Lykesas, School of Physical Education and Sports Science, Aristotle University of Thessaloniki, Greece. E-mail: glykesas@phed.auth.gr

a Afroditi Lola, School of Physical Education and Sports Science, Aristotle University of Thessaloniki, Greece. E-mail: afroditelola@yahoo.gr

\begin{abstract}
The purpose of this study was to evaluate the effects of a Greek traditional dance programme on sensorimotor synchronization (SMS) and reaction time on primary school children (6.41 $\pm .41 \mathrm{yr}$.). We randomly assigned 61 children to either the dance group (31 children, 15 boys, 16 girls, $6.42 \pm$ $.40 \mathrm{yr}$.), who took part in a dance programme of 12 lessons (3 times/week), or the control group (30 children, 16 boys, 14 girls, $6.40 \pm .42 \mathrm{yr}$.). Before and after the intervention, we assessed both groups for SMS (walking in synchrony to 80 and 120 beats/min) and reaction time. Two-way ANOVA repeated measures show that the dance group performed significantly better on SMS compared to the control group at 80 beats/min (BPM), but not at 120 BPM. We discuss the results in relation to spontaneous motor tempo and the information-processing theory. In relation to reaction time, no significant difference was observed between the two groups at the end of the intervention. However, the dance group showed a significant improvement between pre- and post-tests. It may be concluded that a dance programme of 12 lessons constitutes an effective and enjoyable activity for the improvement of young children's sensorimotor synchronization at 80 BPM and perhaps reaction time.
\end{abstract}

\section{Keywords:}

Dance, Sensorimotor Synchronization, Reaction Time, Primary School

\section{Introduction}

The word 'rhythm' originally derives from the Greek word 'rhythmos', which refers to any regular recurring motion. Natural rhythms, such as the rising of the sun or the changing of the seasons affect everything we do, our very existence. For example, circadian rhythms create a regular sleep and eating pattern, which is associated with external environmental and social factors, even in the absence of daily environmental signals (Roenneberg \& Merrow, 2016). Moving on time to a rhythm, usually to music, is an integral part of the human experience and is observed in a wide range of cultures. Although there are many features that 


\section{iejee}

distinguish dance traditions, rhythm is at the centre of almost all of them (Bose et al., 2019).

Sensorimotor synchronization (SMS) refers to the ability to synchronising movements with rhythmic stimuli (Repp \& Su, 2013). Specialists from the field of dance education might be more familiar with the term rhythmic ability (Chatzopoulos et al., 2019). Previous research has documented the importance of SMS for sports performance (MacPherson et al., 2009) and children's language and literacy development (Thomson \& Goswami, 2008). Moreover, poor SMS performance is associated with speech- and writingrelated developmental disorders (Ladányi et al., 2020). Slater et al. (2013) reported that SMS is related to reading ability not only in populations with reading difficulties but in typically-developing children as well. However, despite the importance of SMS for children's motor and literacy development, there is limited research examining the improvement of SMS in school settings.

One of the main skill components of dance is moving in synchrony to the beat of the music. Therefore, dance lessons can be an instrument to improve rhythm. However, there has been limited empirical research on dance programmes integrated into school curricula and their effects on children's SMS improvement (Chatzihidiroglou et al., 2018). Theoretically, dance may play a role in developing children's rhythm, particularly since several studies have reported that professional adult dancers show better synchronization to the beat compared to non-dancers (Jin et al., 2019; Karpati et al., 2016). However, these studies provide a correlational link between dance level and rhythm performance rather than a cause-and-effect link (Chatzopoulos, 2019). Better rhythm performance of the dancers might be attributed to their better sense of rhythm or might be a result of the selection process by experienced dance teachers. Most of the studies examining the effects of dance on children's development have concentrated on dependent variables that are not directly related to dance. For example, previous studies have claimed that dancing may improve children's health-related quality of life (Theocharidou et al., 2018), cardiovascular fitness (Burkhardt \& Brennan, 2012), or contribute to healthy weight maintenance (Staiano et al., 2017). While these variables are desirable educational benefits, they might be achieved through other aerobic activities. Moreover, they do not provide information on whether children's SMS is improved through dance, or how many dance lessons are required for the improvement of SMS.

Previous studies originating from the music education domain have reported positive results of music training on SMS (Repp \& Su, 2013). For example, Zachopoulou et al. (2003) reported that a music and movement programme, based on Orff's approach, improved rhythmic synchronization in preschool children. While music education uses both (a) body movement in synchronization with music and (b) practising musical instruments to improve rhythmic synchronization, dance education involves only body movement in synchrony with rhythmic stimuli. Moreover, music training generally focuses on producing sound using specific hand and finger movements while dance training focuses on following sound using whole-body movements. So far, it has not been fully understood whether dance training affects children's SMS (Jin et al., 2019).

Traditional dance is considered a form of cultural expression that combines physical activity, social interaction, personal and emotional expression. All these elements comprise a holistic experience, which provides pleasure to participants and increases their motivation for participation. This type of dance is relied on structured movement patterns performed in time to rhythmic stimuli and provides opportunities for self-expression. Especially in Greece, folk dances are an integral part of the society and are performed at every important moment of life (e.g., weddings and birthdays). Several studies have reported the psychosocial benefits of traditional Greek dance (Lykesas et al., 2017). However, there is a research gap regarding the effect of traditional dance on young children's SMS.

Reaction time is defined as the time between stimulus onset and the completion of a movement (e.g., pressing of a target button). Performing dance movements often requires fast movement responses to sounds (i.e., well-developed reaction-time ability). Usually, dancers must quickly change their movement or direction in accordance with certain accents in music. An accent is the stress or special emphasis placed on a particular note. An example of an accent would be a louder beat in a song. Accents are important for the beginning of the counting in a dance movement sequence, and the regular accentuation of metrically organised music facilitates the execution of the movements. In dance lessons, teachers often use numbers to count the sequence of dance movements, and the children perform the movements in time with the counting. Young children may become more aware of accents with dance games, such as when dancing around the room and changing direction or movement on accented beats. Kattenstroth et al. (2013) reported that elderly people (mean age of $68.60 \pm 1.45 \mathrm{yr}$.) engaged in dancing showed enhanced reaction time compared to non-dancers. Although reaction time is an important component of the dance performance, the research in this field is limited.

Movement synchronization to rhythmic stimuli and reaction time are crucial factors in dance 
performance and daily/ sports activities (Mann et al., 2007). Traditional dancing requires both SMS and reaction time. Therefore, this study aimed to investigate whether 12 dance lessons would be sufficient to elicit significant improvements in SMS and reaction time of young children.

\section{Method}

\section{Participants}

The study sample consisted of 61 children who attended an primary school centre (31 boys and 30 girls, age $6.41 \pm .41 \mathrm{yr}$.). The dance group consisted of 31 children ( 15 boys and 16 girls, age $6.42 \pm .40 \mathrm{yr}$.) and the control group consisted of 30 children (16 boys and 14 girls, age $6.40 \pm .42 \mathrm{yr}$.). The sample size was determined using G*Power (version 3.1.7, F. Faul, University Kiel, Germany) with a medium effect size at $f=0.25$, an alpha of 0.05 , and a power of 0.80 . Thus, the required sample size was set to 34 (Faul et al., 2007).

We conducted the study in accordance with the ethical guidelines of the local university, and all procedures followed the latest version of the Declaration of Helsinki. Informed consent was obtained from the guardians of the subjects, and the latter could withdraw from the study at any time without any negative consequences. The subjects were free of acute musculoskeletal injuries, did not receive any previous dance or sports training and had no diagnosed learning disabilities.

\section{Procedures}

A physical education (PE) teacher who was also a certified traditional dance instructor taught the dance group. The dance group followed a 4-week dance programme, which took place three times a week (total 12 sessions, $45 \mathrm{~min} /$ session).

The dance programme consisted of the following three traditional dances: 'sousta Kritis', 'ta koukia' ('beans'), and 'choros sta tria'. The dances were chosen according to the following criteria: (a) they consist of basic locomotor movements that can be performed by the children and (b) they are highly repetitive (Little \& Hall, 2017).

The focus of each lesson was one or two of the following dances:

- Sousta. 'Crete Sousta': Men and women perform this dance in pairs. It is a kind of opposite-pair dance. The couples sometimes approach each other, move away from each other, and sometimes hold hands, making turns (https:// www.youtube.com/watch?v=5zOzIETVoT8).

- Ta koukia ('the broad beans'): This is a mimetic dance and it is usually performed on Halloween and at weddings. The dance consists of two parts. In the first, we have a dance with three steps; in the second, we have mimetic movements that represent the planting, carving, picking, and loading of beans (https:// www.youtube.com/watch?v=ZMLbHzavDaA).

- Choros sta tria ('three-step dance'). Choros sta tria is the first dance that a dancer learns from childhood. The dance got its name from the three main progressive steps performed by dancers (https://www.youtube.com/ watch? $v=$ eRAExkVM_FA).

To promote children's self-expression and improvisation, the dance steps and positions were combined with the Laban movement concepts: body awareness, spatial awareness, effort, and relationships (Gilbert, 2015). To foster freedom of choice and increase motivation, the children were explored their own ways of expressing the dance themes, and instructions were provided through analogies (Chatzopoulos et al., 2020; McCutchen, 2006). The structure of the lessons included:

- Warm-up with dynamic stretching exercises and music (Chatzopoulos et al., 2015; Lykesas et al., 2020).

- Introduction of the dance theme and the dance steps.

- Practising the dance steps.

- Dance improvisation; searching for personal expression.

Cooling down with motion and relaxation rhythmic games. Participants in the control group attended their regular $\mathrm{PE}$ lessons during the 4-week intervention period and were taught basic movement skills. The control group was taught by their regular PE teacher.

\section{Measurements}

Before and after the intervention, we applied the following tests to measure SMS and Auditory reaction time of children.

\section{Sensorimotor Synchronization}

We assessed SMS by using the K-Rhythm-test, which consists of a software programme and two force plates (KINVENT, wWW.k-invent.com). The participants were asked to walk in place in synchrony with the beat of the metronome at 80 and 120 beats/minute (BPM) (Karpati et al., 2016). The system captured (a) the time of the beat and (b) the participants' corresponding foot contact (sampling frequency 100 $\mathrm{Hz}$ ). The sensorimotor asynchrony (rhythmic ability) is defined as the temporal absolute difference between the beat and the corresponding foot contact (Karpati et al., 2016). Thus, lower absolute asynchrony scores indicate better performance (i.e., greater synchrony). 


\section{iejee}

Before the measurement, the participants were acquainted with the apparatus and thereafter performed 24 steps in synchrony with the beat of the metronome. The first eight steps of the 24 served as an adaptation period. The mean of the absolute differences from the rest of the 16 steps was used for statistical analysis. The reliability was reported in Chatzopoulos (2019).

\section{Auditory Reaction Time}

The reaction time measurement entailed movement of the dominant foot in response to an auditory stimulus. The participant stands at attention and, upon hearing a sound from the computer (after a fore period of 2-3 sec randomised across trials), performs a fast sidestep of the dominant foot to the corresponding force plate (20-cm distance, Fig. 1). The reaction time is defined as the time between the onset of the stimulus and the participant's foot contact on the force plate. Participants completed one practice attempt and three trials with 30 -second rest periods. We then used the best of the three attempts for statistical analysis. The reliability of the test was reported in Chatzihidiroglou et al. (2018).

\section{Figure 1}

Reaction-Time Measurement
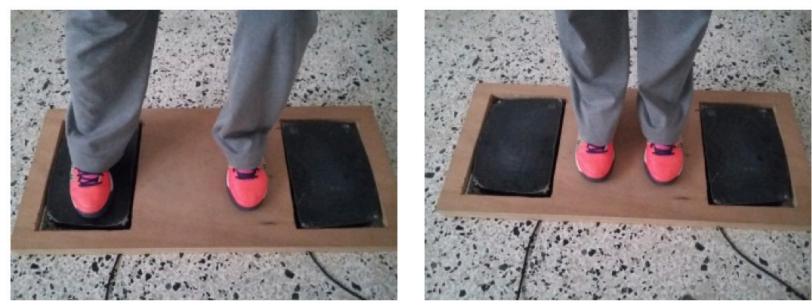

Note. The person in the figure is a model who did not participate in the study.

\section{Statistical Analyses}

We analysed the data using a two-way ANOVA repeated measures design with the between-subject factor group (dance group vs. control group), and the within-subject factor time (pre vs. post). In the case of significant interaction, we applied a post hoc analysis with Bonferroni correction to determine significant differences.

\section{Table 1}

Sensorimotor Synchronization (SMS) (msec) and Auditory Reaction Time (msec) of the Dance Group and the Control Group in Pre- and Post-Tests.

\begin{tabular}{|c|c|c|c|c|}
\hline & \multicolumn{2}{|c|}{ Dance group } & \multicolumn{2}{|c|}{ Control group } \\
\hline & Pre & Post & Pre & Post \\
\hline SMS 80 BPM & $182.8 \pm 48.59$ & $145.4 \pm 48.46^{*}$ & $176.0 \pm 39.59$ & $171.4 \pm 48.78$ \\
\hline SMS 120 BPM & $147.2 \pm 40.92$ & $129.0 \pm 36.46$ & $155.4 \pm 43.96$ & $147.0 \pm 48.22$ \\
\hline Reaction time & $808.1 \pm 295.80$ & $644.1 \pm 227.04$ & $718.4 \pm 306.24$ & $654.5 \pm 187.78$ \\
\hline
\end{tabular}

*Significant difference between the two groups $(p<.05)$.
Furthermore, effect sizes of ANOVA are presented as partial eta-squared values and for t-tests as Cohen's d values. All statistical analyses were performed through SPSS. The statistical significance level was set at $p \leq .05$.

\section{Results}

The descriptive statistics of the dependent variables are presented in Table 1.

\section{Synchronization at 80 beats}

The repeated measures ANOVA indicate a significant interaction effect between group and time of measurement $\left(F_{1,59}=4.38, p=.04, \eta_{p}^{2}=.06\right)$. At the beginning, no significant differences were observed between the two groups $(t=1.1, p=.54, d=.28)$. At the end, the dance group performed significantly better than the control group $(t=2.92, p=.04, d=.75)$.

Synchronization at 120 beats

No significant interaction effect was observed between group and time of measurement $\left(F_{1,59}=.76\right.$, $\left.p=.38, \eta_{p}{ }^{2}=.01\right)$. Moreover, there was no significant difference between the two groups at the beginning $(t=.94, p=.45, d=.24)$ and at the end of the intervention $(t=1.84, p=.10, d=.47)$.

\section{Auditory reaction time}

No significant interaction effect was observed between group and time of measurement $\left(F_{1,59}=1.46\right.$, $\left.p=.23, \eta_{p}{ }^{2}=.02\right)$. There was no significant difference $.25, d=.30)$ and at the end of the intervention ( $t=.19$, $p$ $=.84$ ). However, the dance group showed significant improvement between pre and post measurement ( $t=2.93, p=.006, d=.62)$ whereas the control group showed no significant difference between pre and post measurement $(t=1.03, p=.30, d=.25)$.

\section{Discussion}

SMS and reaction time are crucial factors for the successful execution of daily and sports activities (MacPherson et al., 2009). The purpose of this study was to investigate the effects of a Greek traditional dance programme on SMS and reaction time of primary between the two groups at the beginning $(t=.01, p=$ 
school children. The study results show that a dance programme of 12 lessons may enhance children's rhythmic synchronization at 80 BPM but not at 120 BPM. Moreover, regarding reaction time, no significant differences was observed between the dance group and control group at the end of the intervention.

The ability of movement synchronization with music is an important aspect of dance performance. In dance lessons, movement synchronization refers to either the synchronization of one's movements with the beat of music or with those of another dance partner. The results of this study show that a Greek traditional dance programme enhanced the children's SMS at 80 beats $/ \mathrm{min}$. This finding is in agreement with those originating from the field of music/movement education (Zachopoulou et al., 2003). However, while music/movement education focuses on producing sounds with hand and finger rhythmic movements, dance training focuses on aesthetic whole-body movements in synchrony with the beat of the music. Therefore, a comparison of the results of studies in music education and those of the present study should be made with caution. Besides, the absence of other studies necessitates further investigation regarding the effects of dance on rhythmic synchronization.

Previous studies have shown that professional dancers demonstrate better synchronization performance than non-dancers (Jin et al., 2019; Karpati et al., 2016). However, these studies do not provide a causal link between the dancers' level and rhythmic synchronization. Therefore, it is unclear whether the dancers' superior performance is the result of extensive dance training or the result of the selection process by experienced dance teachers. The participants of this study were young children without any previous music or dance experience. Therefore, the improvement of SMS could be an indication that it may be trained in young children.

According to Drewing et al. (2006), rhythmic synchronization substantially improves during childhood until about the late teens, and thereafter remains at least relatively stable until old age. According to the current study, a dance programme enhanced the SMS of young children. Therefore, future studies could examine whether dancing increases the rate and the magnitude of the developmental trend of children's SMS.

An important issue of this study is the absence of significant difference between the dance group and the control group at 120 BPM. The non-significant finding might be attributed to children's Spontaneous Motor Tempo (SMT), which refers to the rate at which children choose to tap in the absence of external rhythmic stimuli. Studies of beat synchronization in children reported that approximately 120 BPM is the preferred spontaneous tempo of children (McAuley et al., 2006). Additionally, it has been shown that movement synchronization is most accurate when the tempo of the rhythmic stimulus is around the SMT (Styns et al., 2007); thus, any further improvement may be difficult to achieve. Moreover, the period of 12 dance lessons may not be sufficient to improve synchronization at 120 BPM. The limited number of studies in this area necessitates further research.

SMS is an essential component of the perception and performance of dance movements and daily/ sports activities. Yet, the underlying mechanisms are still not entirely understood. Two main theoretical approaches to SMS can be distinguished: information-processing theory and dynamic systems theory. Informationprocessing theory usually deals with discrete movements (i.e., those preceded and followed by a period without motion) whereas dynamic theory deals primarily with continuous movement. The basic mechanisms of SMS are studied with the fingertapping paradigm. As finger-tapping on a surface generates discrete events, most researchers using the finger-tapping paradigm have adopted informationprocessing perspective whereas dynamic systems theorists generally preferred continuous movement tasks (Repp \& Su, 2013).

According to the information-processing theory, rhythmic synchronization of discrete movements is thought to involve a clock-like mechanism that incorporates an explicit representation of the time interval defined by each discrete movement.

It is assumed that synchronization with an external rhythm is achieved using a central timekeeper through three distinct processes: (a) an internal clock that captures temporal information and acts as a pacemaker, (b) relational memory systems that estimate duration and create a memory trace of the time interval, and (c) decision processes to enable motor preparation and trigger action initiation (Rose et al., 2021). Within the theoretical framework of the information-processing approach, a stable SMS is maintained through the processes of phase and period correction. Without such correction, error variance due to internal timekeeper variability would accumulate and lead to asynchrony (Repp \& Su, 2013). In the phase-correction process, information about the asynchrony (time difference) between the finger-tap and the click of the metronome is used to adjust the start point of the next timekeeper interval. It is assumed that this is an automatic process (i.e., it occurs without participants' awareness). The period correction adjusts the internal representation of the temporal interval when mismatch is detected between an inter-tap (period between two finger- 


\section{iejee}

taps) and metronome intervals (period between two clicks of the metronome). The period-correction process is assumed to be more under cognitive control. Phase correction is believed to be sufficient for maintaining synchronization with an isochronous stimulus sequence (such as that produced by a metronome) whereas period correction appears to come into play only when the period of the stimulus sequence is changed in a systematic way (e.g., tempo changes) (Repp \& Su, 2013).

In dance lessons, participants often must quickly change their movements in time with certain music accents. Therefore, it was expected that a dance programme would improve their auditory reaction time. According to the study, there was no significant difference between the dance group and the control group at the end of the intervention. Also, the dance group performed significantly better at the end of the intervention compared to pre-test data. This could indicate that dance activities may have a positive effect on the development of reaction time. The findings of this study are aligned with those of a previous study involving preschool children and that reported no significant difference between the dance group and the control group at the end of a dance programme (Chatzihidiroglou et al., 2018). On the contrary, Kattenstroth et al. (2013) reported that the reaction time of elderly participants significantly improved after a dance programme. The different findings could be attributed to the different ages of the participants of the samples. The sample of Kattenstroth et al. (2013) consisted of elderly people, ours of young children, and it is well established that the reaction time performance decreases and becomes more variable with age (Dykiert et al., 2012). Furthermore, physical activity of older participants also affects the reaction-time performance (Spirduso, 1980). Therefore, the comparison of the results of studies with samples of participants of different ages should be made cautiously. Nonetheless, there is definitely a need for further research on the effects of dance on children's reaction time.

\section{Limitation}

The study has some limitations. The limitation of this study is that the study participants consisted of young children (6-7 years old). SMS and auditory reaction time measurements require concentration that young children probably did not have at the time of measurement.

\section{Conclusion}

SMS and auditory reaction time are important factors for the successful execution of daily and sports activities. In the present study, the importance of dancing for the development of SMS in young children has been stressed. Considering the importance of SMS for children's motor and cognitive development, we suggest that dance should be a basic component of the curriculum in primary school education.

\section{Acknowledgements}

The authors thank the children for their participation in the study.

\section{References}

Bose, A., Byrne, Á., \& Rinzel, J. (2019). A neuromechanistic model for rhythmic beat generation. PLOS Computational Biology, 15(5), e1006450.

Burkhardt, J., \& Brennan, C. (2012). The effects of recreational dance interventions on the health and well-being of children and young people: A systematic review. Arts \& Health, 4(2), 148-161.

Chatzihidiroglou, P., Chatzopoulos, D., Lykesas, G., \& Doganis, G. (2018). Dancing effects on preschoolers' sensorimotor synchronization, balance, and movement reaction time. Perceptual and Motor Skills, 125(3), 463-477.

Chatzopoulos, D. (2019). Effects of ballet training on proprioception, balance, and rhythmic synchronization of young children. Journal of Exercise Physiologyonline, 22(2), 26-37.

Chatzopoulos, D., Yiannakos, A., Kotzamanidou, M., \& Bassa, E. (2015). Warm-up protocols for high school students. Perceptual and Motor Skills, 121(1), 1-13.

Chatzopoulos, D., Doganis, G., \& Kollias, I. (2019). Effects of creative dance on proprioception, rhythm and balance of preschool children. Early Child Development and Care, 189(12), 1943-1953.

Chatzopoulos, D., Foka, E., Doganis, G., Lykesas, G., \& Nikodelis, T. (2020). Effects of analogy learning on locomotor skills and balance of preschool children. Early Child Development and Care, $1-9$.

Drewing, K., Aschersleben, G., \& Li, S.-C. (2006). Sensorimotor synchronization across the life span. International Journal of Behavioral Development, 30(3), 280-287.

Dykiert, D., Der, G., Starr, J. M., \& Deary, I. J. (2012). Sex differences in reaction time mean and intraindividual variability across the life span. Developmental Psychology, 48(5), 1262-1276. 
Faul, F., Erdfelder, E., Lang, A.-G., \& Buchner, A. (2007). G*Power 3: A flexible statistical power analysis program for the social, behavioral, and biomedical sciences. Behavior Research Methods, 39, 175-191.

Gilbert, A. G. (2015). Creative dance for all ages: A conceptual approach (Second edition). Human Kinetics.

Jin, X., Wang, B., Lv, Y., Lu, Y., Chen, J., \& Zhou, C. (2019). Does dance training influence beat sensorimotor synchronization? Differences in finger-tapping sensorimotor synchronization between competitive ballroom dancers and nondancers. Experimental Brain Research, 237(3), 743-753.

Karpati, F. J., Giacosa, C., Foster, N. E. V., Penhune, V. B., \& Hyde, K. L. (2016). Sensorimotor integration is enhanced in dancers and musicians. Experimental Brain Research, 234(3), 893-903.

Kattenstroth, J.-C., Kalisch, T., Holt, S., Tegenthoff, M., \& Dinse, H. R. (2013). Six months of dance intervention enhances postural, sensorimotor, and cognitive performance in elderly without affecting cardio-respiratory functions. Frontiers in Aging Neuroscience, 5, 5.

Ladányi, E., Persici, V., Fiveash, A., Tillmann, B., \& Gordon, R. L. (2020). Is atypical rhythm a risk factor for developmental speech and language disorders? Wiley Interdisciplinary Reviews. Cognitive Science, 11(5), e1528.

Little, S., \& Hall, T. (2017). Selecting, Teaching and Assessing Physical Education Dance Experiences. Journal of Physical Education, Recreation \& Dance, 88(3), 36-42.

Lykesas, G., Giosos, I., Theocharidou, O., Chatzopoulos, D., \& Koutsouba, M. (2017). The effect of a traditional dance program on health-related quality of life as perceived by primary school students. Journal of Education and Training Studies, 6(1), 96.

Lykesas, G., Giossos, I., Chatzopoulos, D., Koutsouba, M., Douka, S., \& Nikolaki, E. (2020). Effects of Several Warm-Up Protocols (Static, Dynamic, No Stretching, Greek Traditional Dance) on Motor Skill Performance in Primary School Students. International Electronic Journal of Elementary Education, 12(5), 481-487.
MacPherson, A. C., Collins, D., \& Obhi, S. S. (2009). The Importance of Temporal Structure and Rhythm for the Optimum Performance of Motor Skills: A New Focus for Practitioners of Sport Psychology. Journal of Applied Sport Psychology, 21(sup1), S48-S61.

Mann, D. T. Y., Williams, A. M., Ward, P., \& Janelle, C. M. (2007). Perceptual-cognitive expertise in sport: A meta-analysis. Journal of Sport \& Exercise Psychology, 29(4), 457-478.

McAuley, J. D., Jones, M. R., Holub, S., Johnston, H. M., \& Miller, N. S. (2006). The time of our lives: Life span development of timing and event tracking. Journal of Experimental Psychology. General, 135(3), 348-367.

McCutchen, B. P. (2006). Teaching dance as art in education. Human Kinetics.

Repp, B. H., \& Su, Y.-H. (2013). Sensorimotor synchronization: A review of recent research (2006-2012). Psychonomic Bulletin \& Review, 20(3), 403-452.

Roenneberg, T., \& Merrow, M. (2016). The Circadian Clock and Human Health. Current Biology: $C B$, 26(10), R432-43.

Rose, D., Ott, L., Guérin, S. M. R., Annett, L. E., Lovatt, P., \& Delevoye-Turrell, Y. N. (2021). A general procedure to measure the pacing of body movements timed to music and metronome in younger and older adults. Scientific Reports, 11(1), 3264.

Slater, J., Tierney, A., \& Kraus, N. (2013). At-risk elementary school children with one year of classroom music instruction are better at keeping a beat. Plos One, 8(10), e77250.

Spirduso, W. W. (1980). Physical fitness, aging, and psychomotor speed: A review. Journal of Gerontology, 35(6), 850-865.

Staiano, A. E., Marker, A. M., Beyl, R. A., Hsia, D. S., Katzmarzyk, P. T., \& Newton, R. L. (2017). A randomized controlled trial of dance exergaming for exercise training in overweight and obese adolescent girls. Pediatric Obesity, 12(2), 120-128

Styns, F., van Noorden, L., Moelants, D., \& Leman, M. (2007). Walking on music. Human Movement Science, 26(5), 769-785. 
Theocharidou, O., Lykesas, G., Giossos, I., Chatzopoulos, D., \& Koutsouba, M. (2018). The positive effects of a combined program of creative dance and BrainDance on health-related quality of life as perceived by primary school students. Physical Culture and Sport. Studies and Research, 79(1), 42-52.

Thomson, J. M., \& Goswami, U. (2008). Rhythmic processing in children with developmental dyslexia: Auditory and motor rhythms link to reading and spelling. Journal of Physiology, Paris, 102(1-3), 120-129.

Zachopoulou, E., Derri, V., Chatzopoulos, D., \& Ellinoudis, T. (2003). Application of Orff and Dalcroze activities in preschool children: Do they affect the level of rhythmic ability? Physical Educator, $60(2), 50-66$. 